\title{
The rare reason of pain in hip girdle: Mucolipidosis type 3 gamma
}

\author{
Abdulkerim Kolkıran ${ }^{1 \oplus}$, Beren Karaosmanoğlu ${ }^{2 \oplus}$, Zihni Ekim Taşkıran² ${ }^{2 \oplus}$ \\ Pelin Özlem Şimşek Kiper ${ }^{1 \oplus}$, Gülen Eda Utine ${ }^{1 \oplus}$ \\ ${ }^{1}$ Division of Pediatric Genetics, Department of Pediatrics and ${ }^{2}$ Department of Medical Genetics, Hacettepe University Faculty of \\ Medicine, Ankara, Turkey.
}

\begin{abstract}
Background. Mucolipidosis type 3 gamma (ML-III $\gamma$ ) is an autosomal recessive, rare and slowly progressive lysosomal storage disease. Short stature, restricted joint mobility, thick skin, and flat face with mildly coarse features are major clinical findings. It usually manifests in the third year. With advancing age, claw hand deformities, carpal tunnel syndrome, and scoliosis may develop. Morbidity is determined mainly by skeletal involvement. N-acetyl glucosamine- 1 phospotransferase enzyme is composed of $2 \alpha, 2 \beta$ and $2 \gamma$ subunits. The active enzyme is essential in the transport of hydrolases to the lysosomes, via addition of mannose-6-phosphate in the Golgi apparatus. GNPTG gene encodes the $\gamma 2$ subunits, and biallelic mutations cause ML-III $\gamma$.
\end{abstract}

Case. A previously healthy 14-year-old male patient had leg pain after the age of nine, and was admitted with short stature, mild coarse face, pectus deformity, digital stiffness, scoliosis, genu valgum and mitral valve prolapse. He did not have intellectual disability or corneal clouding. Radiographs showed irregularities in the acetabular roof and proximal epiphyses of the femur and irregularities in the end plates of vertebral bodies. A novel homozygous missense variant in the exon 5 of GNPTG, c.316G>T, confirmed the diagnosis of MLIII $\gamma$. Juvenile idiopathic arthritis (JIA), progressive pseudorheumatoid dysplasia (PPRD), ML-II, ML-III $\alpha \beta$, galactosialidosis and mucopolysaccharidosis should be considered in the differential diagnosis.

Conclusions. ML-III $\gamma$ should be kept in mind in populations with high consanguineous marriage rates or with possible founder effect, in patients with short stature and skeletal destruction. Genetic tests should be planned for a definitive diagnosis.

Key words: Mucolipidosis type 3 gamma (MLIII $\gamma)$, GNTPG gene, Genetic skeletal disorder, painful hip girdle.

Mucolipidosis type 3 gamma (MLIII $\gamma$ ) is a rare, slowly progressive lysosomal storage disease reported for the first time by Maroteaux and Lamy in 1966. ${ }^{1-5}$ It is inherited in an autosomal recessive (AR) manner. ${ }^{1-5}$ Stiffness in the finger joints usually begins in early childhood, whereas short stature, scoliosis and skeletal deformities are observed as a result of large joint involvement with advancing age. ${ }^{5}$

N-acetyl glucosamine-1 phospotransferase (GlcNAc-PTase) enzyme is a $540-\mathrm{kDa}$ weight

$凶$ Abdulkerim Kolkıran

kolkiran@hotmail.com

Received 8th April 2021, revised 15th March 2021, accepted 21st May 2021. heterohexameric polypeptide composed of $2 \alpha$, $2 \beta$ and $2 \gamma$ subunits. ${ }^{1-9}$ Membrane dependent enzyme precursors $\alpha 2 \beta 2$ subunits and $\gamma 2$ subunits are combined and carried to the Golgi apparatus. ${ }^{4}$ Here, the $\alpha 2 \beta 2$ subunits in the precursor enzyme complex are activated with cleavage by site- 1 protease (S1P). ${ }^{4}$ The active enzyme plays an essential role in the transport of hydrolases to the lysosomes by modifying them with addition of mannose-6-phosphate (M6P) residues in the Golgi apparatus. ${ }^{2-6}$ Hydrolases that cannot be modified with M6P as a result of GlcNAc-PTase enzyme deficiency cannot be transported to lysosomes. ${ }^{1,4,6}$ Hence, non-digestible macromolecules accumulate in lysosomes, as granular intracytoplasmic inclusion bodies that can be 
seen microscopically. ${ }^{1,4,6}$ These hydrolases that cannot be transported to lysosomes are secreted into the intercellular spaces. ${ }^{1,4}$

The GNPTAB gene encodes $\alpha / \beta$ subunits and the GNPTG gene encodes $\gamma 2$ subunits. ${ }^{1,2,4,5,7}$ Homozygous or compound heterozygous mutations in the GNPTG gene, which produce GlcNAc-PTase enzyme $\gamma$ subunit deficiency, cause MLIII $\gamma$ disease. ${ }^{1}$

We report a 14-year-old male patient presenting with the complaints of short stature, scoliosis and hip joint damage.

\section{Case Report}

A 14-year-old male patient was consulted to the genetics department. He had bilateral Perthes disease sequela and scoliosis. He was the third live born to his 25-year-old mother following the third pregnancy, with an uneventful pregnancy at term, with a birth weight of 2600 $\mathrm{g}$, by spontaneous vaginal birth. The parents were consanguineous (first cousins). Two healthy male children, now aged 18 and 15 years old, were born from the first and second pregnancies. The fourth pregnancy of the mother resulted in miscarriage in the 20th week of pregnancy. The grandson of the paternal uncle had similar findings in the hip joint. The pedigree of our patient is shown in Figure 1.

Bilateral Perthes sequelae and scoliosis were detected and femoral osteotomy operation was performed at the age of ten years when he was first referred due to in-toeing and bilateral leg pain. Stiffness of finger joints began at that age. All developmental steps were normal and there was no intellectual disability.

On physical examination, his weight was 39 $\mathrm{kg}$ (3rd-10th centile), height was $145 \mathrm{~cm}(<3 \mathrm{rd}$ centile) and head circumference was $54 \mathrm{~cm}$ (25th-50th centile). Arm span was $154 \mathrm{~cm}$. The upper to lower segment ratio was calculated

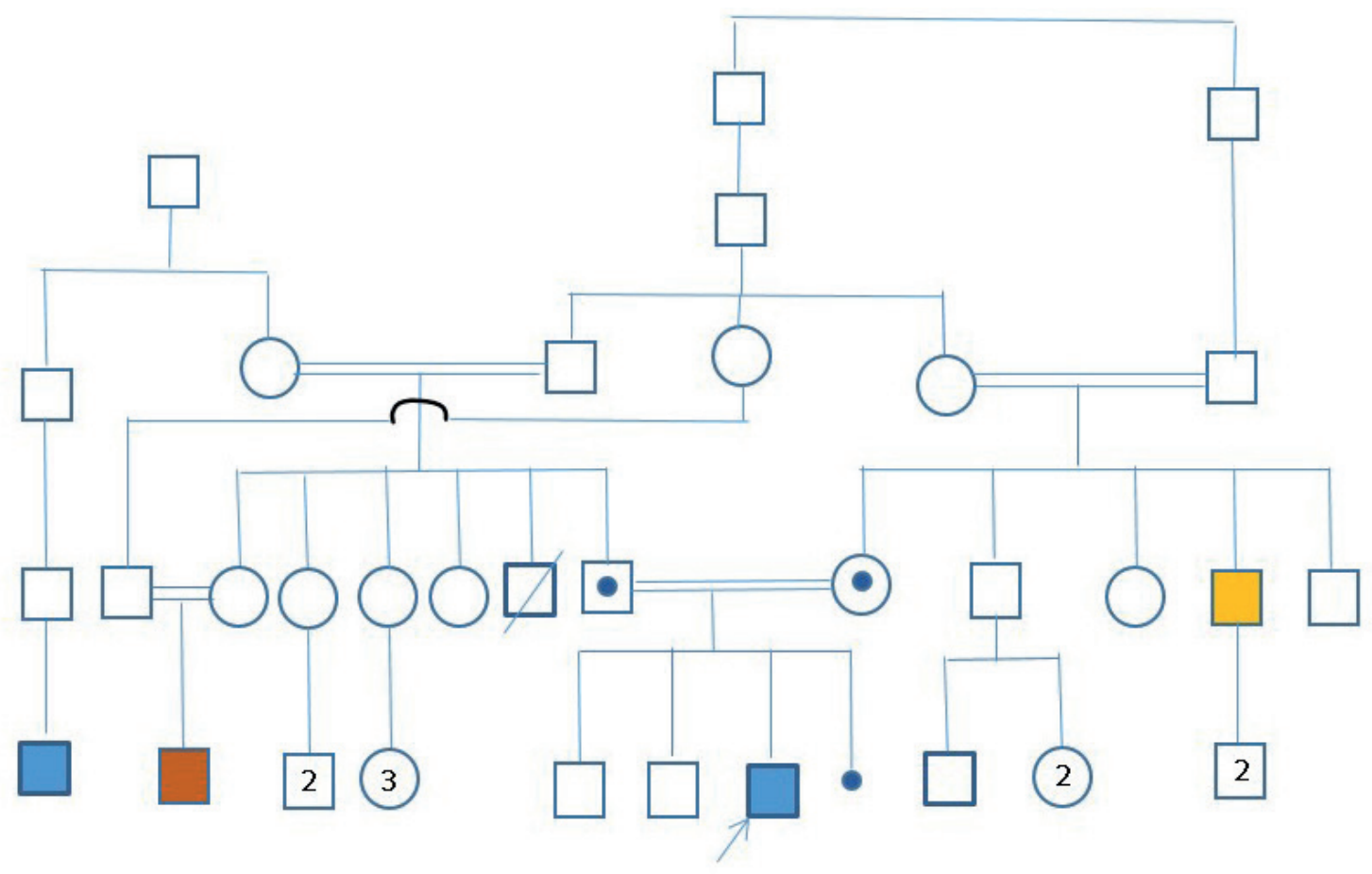

\section{Argininemia $\square$ Hearing loss}

Fig. 1. Pedigrees of the patient. 
as $0.85(<-2 \mathrm{SD}) .{ }^{10}$ Narrow forehead, deep set eyes, bulbous nose tip, dental crowding, short and webbed neck, mild coarse face, pectus carinatum superiorly and excavatum inferiorly, scoliosis, accentuated thoracic kyphosis and lumbar lordosis, stiffness of the fingers, genu valgum and pes planus were noted. Acute phase reactants were all normal. Diseases such as progressive pseudorheumatoid dysplasia (PPRD) and juvenile idiopathic arthritis (JIA) were reviewed and excluded in differential diagnosis. Informed consent was received from the family for this publication.

\section{Genetic analysis}

Genetic analysis was performed after informed consent was taken from the parents. The study protocol was approved by the Hacettepe University Ethics Committee (GO 15-530/25). DNA samples of the patient were subjected to whole exome sequencing (WES) analysis at the Hacettepe University Faculty of Medicine, Pediatric Genetics Laboratory, Ankara (Turkey). Exome libraries were prepared by using the Ion AmpliSeq Exome RDY Kit, and sequencing was performed by Ion Proton System (Thermo Fisher Scientific). Homozygosity mapping and bioinformatics analysis were performed as outlined previously. ${ }^{11}$ Segregation analysis was performed from DNA samples taken from the parents.

\section{Genetic results}

According to the NM_032520.4 coded transcript of the GNPTG in the NCBI database, a novel missense variant c.316G $>$ T (p.Gly106Cys) was detected in exon 5. Parents were heterozygous for the same variant. According to the ACMG 2015 criteria, the variant is "disease causing". The variant is predicted as disease-causing according to in silico databases such as MutationTaster, and the CADD score is 32 .

\section{Discussion}

Clinical manifestations of the extremely rare MLIII $\gamma$ disease is quite variable. ${ }^{12}$ While very mildly affected cases present in later childhood with isolated hip dysplasia and some vertebral changes, in most of the cases MLIII $\gamma$ is a slowly progressive disease. ${ }^{5}$ Increased stiffness of the joints suggestive of idiopathic arthritis is generally noted after the second year of life. . $^{1,3,12}$ Although it is a multisystemic disease, skeletal involvement mainly determines morbidity. ${ }^{5,8}$ Although destruction of the acetabulum and femoral head begin by 5 years of age, this usually begins in the adolescent period. ${ }^{1,3}$ Skeleton findings progress as the age advances. ${ }^{1}$

In addition to dysostosis multiplex findings, progressive osteopenia, osteoarthritis and erosive bone lesions are seen as a result of cartilage and bone destruction in many joints including shoulder, elbow, hip and knee. ${ }^{1,3,5,8}$ Scoliosis, claw hand deformity due to stiffness and contractures in joints and spinal cord compression can be seen particularly with advancing age. ${ }^{1,8}$ Depending on these hip and leg pains, difficulty in squatting and a decrease in walking ability are observed. ${ }^{1}$ Carpal tunnel syndrome is a common finding. ${ }^{1,8}$ The radiographic findings of our patient are summarized in Figure 2.

Although the abnormal findings of hip joint in the previously reported patients generally started in late adolescent period, the present patient had an earlier (9 years old) onset of joint problems and walking difficulty. ${ }^{3}$ Bilateral femoral osteotomy operation was performed at the age of ten years and mild dextroscoliosis, lumbar lordosis, and thoracic kyphosis were detected in the follow up. The patient had pectus carinatum and excavatum deformity.

Patients with the onset of stiffness of fingers at the age of 1.5 years are reported, nevertheless this usually begins in early childhood., ${ }^{1,3}$, Nampoothiri et al. ${ }^{1}$ reported that hand deformity started at the age of 10 years in Turkish patients. Onset of stiffness at the hand fingers at older ages is associated with a milder clinical course. In our patient, the stiffness of the hands began at the age of 12 years. ${ }^{5}$ 

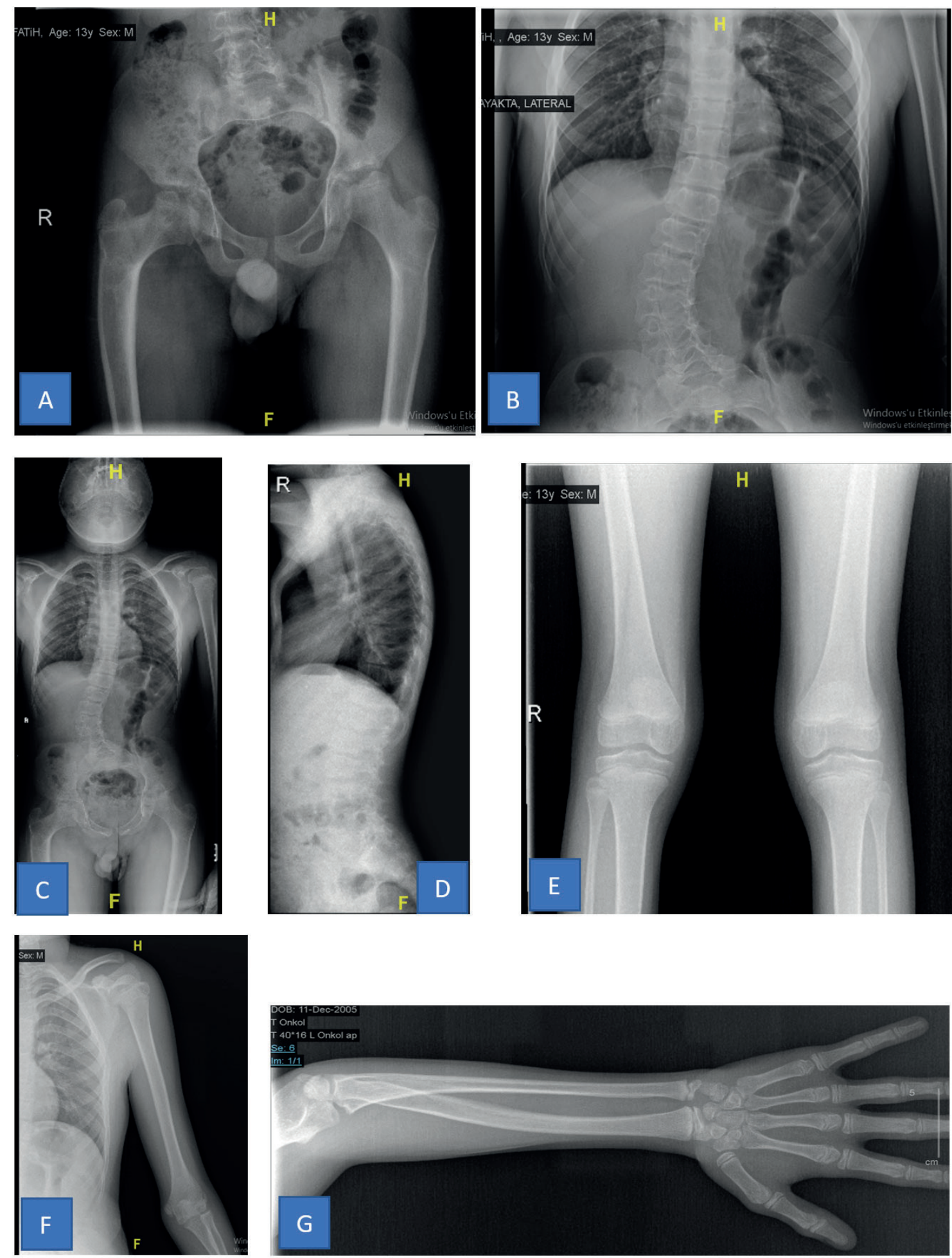

Fig. 2. Radiographs of the patient. A) Pelvic radiograph revealed irregularities in the acetabular roof, epiphyseal dysplasia of the proximal femora, coxa valga, and shallow acetabula. B-C-D) Mild scoliosis is seen on chest and abdomen radiography. Mildly flattened vertebral bodies with irregular upper and lower end plates and irregular narrowing of the intervertebral spaces and hypoplasia of the dorsal parts of the thoracic vertebral bodies and mild anterior hypoplasia is seen radiograph of spine. E-F-G) The long tubuler bones were normal. 
Other clinical features of MLIII $\gamma$ include mild coarse facial appearance, short stature, corneal clouding, mild intellectual disability, restrictive lung disease, and heart valve anomalies..$^{1-3,5,6,8,9}$ Woody skin texture may occur that starts on the back of the hand and forearm and can be seen in the face over time..$^{1-3}$ Mild to moderate intellectual disability or learning disabilities may be seen in almost half of the MLIII patients. ${ }^{12}$ Nevertheless, intellectual disability is usually seen in MLIII $\alpha \beta$ disease but not in MLIII $\gamma$ disease. ${ }^{5}$

There was no woody skin texture or sclerodermalike appearance on the skin of our patient. He had a mild coarse face appearance. Although he had pectus deformity, his lung functions were normal. Motor and language developmental steps were normal and he had no intellectual disability. There was no murmur on physical examination, but mild mitral valve prolapse was detected on echocardiography. There was no corneal clouding in the eye examination.

Besides clinical findings, increase in lysosomal hydrolases in plasma and decrease in intracellular enzyme level in fibroblast cultures support the diagnosis, but for the definitive diagnosis of MLIII $\gamma$, a mutation in the GNTPG gene was detected and verified..$^{1,3,4,8,12}$ The segregation analysis revealed that the parents were heterozygous carriers. Genetic counseling was given accordingly.

GlcNAc-PTase enzyme activity determines the severity and name of the disease. ${ }^{6}$ If mutations in the GNTPAB gene cause complete absence of enzyme activity, it causes MLII disease, while in the presence of $10 \%$ and above enzyme activity occurs MLIII $\alpha \beta$ disease. ${ }^{1,2,4,6}$ Mutations in the GNTPG gene cause MLIII $\gamma$ disease. ${ }^{1,3,9}$ So far, 50 different mutations in the GNTPG gene have been reported in 79 patients. ${ }^{4}$ These mutations occur 35\% frameshift 23\% nonsense and $23 \%$ splice site, $15 \%$ missense, $4 \%$ deletion duplication and insertions. ${ }^{4} 23 \%$ of all mutations are intronic mutations. ${ }^{4}$

Owing to the initial joint findings, patients can be followed up with a diagnosis of JIA, one of the most common chronic diseases of childhood, before the establishment of the definite diagnosis. ${ }^{3}$ Even a number of treatments such as, nonsteroidal anti-inflammatory drugs, systemic and intraarticular glucocorticoids and nonbiologic and biologic disease modifying antirheumatic drugs might have been tried before the patient's admission. Differential diagnosis can be made with high serum lysosomal enzyme activity, presence of dysostosis multiplex findings, lack of high serum acute phase reactants levels, autosomal recessive (AR) inheritance pattern and the detection of a pathogenic GNTPG gene variant. ${ }^{3}$ PPRD resulting from WISP3 gene mutations should be considered in the differential diagnosis due to joint stiffness, kyphoscoliosis and claw hand deformity. ${ }^{3}$ However, PPRD differs by the presence of milder vertebral changes, broad lower ilia, swollen ends of the short tubular bones, normal serum hydrolase levels, and no GNTPG gene mutation.,12 Mucolipidosis II, ML-III $\alpha \beta$, mucopolysaccharidosis types I, II, IV, VI, and VII, other genetic skeletal disorders including spondyloepiphyseal dysplasia congenita, Kniest dysplasia, X-linked spondyloepiphyseal dysplasia tarda, and Dyggve-Melchior-Clausen dysplasia should all be considered in the differential diagnosis. ${ }^{3,12}$ Differential diagnosis is easier in ML-II disease, as it occurs due to the complete absence of GlcNAc-PTase enzyme and the clinical findings begin prenatally or at birth and result in demise in early childhood. ${ }^{3}$ The clinical distinction between MLIII $\gamma$ and ML-III $\alpha \beta$ is quite difficult, nevertheless it is generally accepted that MLIII $\gamma$ is a milder form of mucolipidosis compared to that of MLIII $\alpha \beta$ disease. ${ }^{4,9}$ In addition, forearm hypertrophy and supination defect may be a distinctive feature in MLIII $\gamma$ disease. ${ }^{1}$

There is no cure for MLIII $\gamma$ yet. $^{3}$ Treatment is completely symptomatic and supportive. ${ }^{3}$ Physical therapy can be applied for joint stiffness and contractures. Total hip replacement (THR) and knee replacement therapy can be applied since complaints about hip and knee joints and the associated findings increase with 
advancing age. ${ }^{1,3}$ Surgical correction may be required for carpal tunnel syndrome and claw hand deformities. ${ }^{3}$ For scoliosis and spinal cord compression, supportive treatments and surgical corrections should be applied as necessary. ${ }^{5}$ As the pain in the hip and knee can significantly decrease the quality of life, necessary treatments should be applied. Biphosphonate therapy can be given when necessary for osteoporosis. ${ }^{3,5}$

Despite being a rare disease, the prevalence of ML-III $\gamma$ may be high especially in countries with high consanguineous marriage rates. For this reason, appropriate genetic tests should be planned in patients presenting with joint findings in the hips and fingers, coarse face, short stature, corneal clouding and scoliosis.

\section{Author contribution}

The authors confirm contribution to the paper as follows: study conception and design: AK, PÖŞK, GEU; data collection: AK, BK, ZET, PÖŞK, GEU; analysis and interpretation of results: AK, BK, ZET; draft manuscript preparation: AK, ZET, PÖŞK, GEU. All authors reviewed the results and approved the final version of the manuscript.

\section{Ethical approval}

Informed consent was received from the family for this publication. The study protocol was approved by the Hacettepe University Ethics Committee (GO 15-530/25).

\section{Funding}

This study no funded.

\section{Conflict of interest}

The authors declare no conflict of interest.

\section{REFERENCES}

1. Nampoothiri S, Elcioglu NH, Koca SS, et al. Does the clinical phenotype of mucolipidosis-III $\gamma$ differ from its $\alpha \beta$ counterpart?: supporting facts in a cohort of 18 patients. Clin Dysmorphol 2019; 28: 7-16. https://doi. org/10.1097/MCD.0000000000000249

2. Khan MA, Hussain A, Sher G, Zubaida B, Naeem M. Genetic testing of a large consanguineous Pakistani family affected with mucolipidosis III gamma through next-generation sequencing. Genet Test Mol Biomarkers 2018; 22: 541-545. https:/doi. org/10.1089/gtmb.2018.0123

3. Raas-Rothschild A, Spiegel R. Mucolipidosis III gamma. In: Adam MP, Ardinger HH, Pagon RA, et al. (eds). GeneReviews ${ }^{\circledR}$. Seattle, WA: University of Washington, 2010.

4. Velho RV, Harms FL, Danyukova T, et al. The lysosomal storage disorders mucolipidosis type II, type III alpha/beta, and type III gamma: update on GNPTAB and GNPTG mutations. Hum Mutat 2019; 40: 842-864. https://doi.org/10.1002/humu.23748

5. Tüysüz B, Kasapçopur Ö, Alkaya DU, Şahin S, Sözeri B, Yeşil G. Mucolipidosis type III gamma: three novel mutation and genotype-phenotype study in eleven patients. Gene 2018; 642: 398-407. https://doi. org/10.1016/j.gene.2017.11.052

6. van Meel E, Kornfeld S. Mucolipidosis III GNPTG missense mutations cause misfolding of the $\gamma$ subunit of GlcNAc-1-phosphotransferase. Hum Mutat 2016; 37: 623-626. https://doi.org/10.1002/ humu.22993

7. Braulke T, Pohl S, Storch S. Molecular analysis of the GlcNac-1-phosphotransferase. J Inherit Metab Dis 2008; 31: 253-257. https://doi.org/10.1007/s10545008-0862-5

8. Oussoren E, van Eerd D, Murphy E, et al. Mucolipidosis type III, a series of adult patients. J Inherit Metab Dis 2018; 41: 839-848. https://doi. org/10.1007/s10545-018-0186-Z

9. Liu S, Zhang W, Shi H, Meng Y, Qiu Z. Three novel homozygous mutations in the GNPTG gene that cause mucolipidosis type III gamma. Gene 2014; 535: 294-298. https://doi.org/10.1016/j.gene.2013.11.010

10. Gripp KW, Slavotinek AM, Hall JG, Allanson JE. Handbook of Physical Measurements (3rd ed). New York: Oxford University Press, 2007: 240-244.

11. Utine GE, Taşkıran EZ, Koşukcu C, et al. HERC1 mutations in idiopathic intellectual disability. Eur J Med Genet 2017; 60: 279-283. https://doi. org/10.1016/j.ejmg.2017.03.007

12. Spranger JW, Brill PW, Hall C, Nishimura G, SupertiFurga A, Unger S. Bone Dysplasias: An Atlas of Genetic Disorders of Skeletal Development (4th ed). New York: Oxford University Press, 2018: 129-130. https://doi.org/10.1093/med/9780190626655.001.0001 